Revue pluridisciplinaire d'études médiévales

\title{
Comptes féminins, contes du quotidien ? Les activités des duchesses de la seconde Maison d'Anjou à la lumière de leurs finances
}

\section{Marion Chaigne}

\section{(2) OpenEdition}

\section{Journals}

Édition électronique

URL : http://journals.openedition.org/questes/4114

DOI : 10.4000/questes.4114

ISSN : 2109-9472

Éditeur

Les Amis de Questes

\section{Édition imprimée}

Date de publication : 15 septembre 2008

Pagination : 64-81

ISSN : $2102-7188$

\section{Référence électronique}

Marion Chaigne, «Comptes féminins, contes du quotidien? Les activités des duchesses de la seconde Maison d'Anjou à la lumière de leurs finances », Questes [En ligne], 15 | 2008, mis en ligne le 01 janvier 2014, consulté le 24 avril 2019. URL : http://journals.openedition.org/questes/4114 ; DOI : 10.4000 /questes.4114 


\section{Comptes féminins, contes du quotidien?}

\section{Les activités des duchesses de la seconde Maison d'Anjou à la lumière de leurs finances}

\section{Marion CHAIGNE}

S'intéresser aux activités quotidiennes des femmes de la fin du Moyen Âge nécessite une réflexion préalable sur ces termes. Le terme d'activitas apparait en 1425. Il désigne la puissance d'agir, autrement dit une qualité, une vertu attachée à celui qui la possède. D'emploi plus concret, il caractérise également la mise en exercice de cette puissance d'agir. Tautologiquement, l'état agissant d'un individu est donc le propre d'un sujet actif. Or, les femmes sont communément décrites, tant dans la littérature médiévale que dans l'historiographie contemporaine, comme des êtres passifs, proies des règles imposées par un contrôle masculin - souvent clérical - et objets d'échange dans les stratégies familiales et sociales ${ }^{1}$. Ces considérations sont souvent systématisées lorsque les sujets en question appartiennent aux familles princières. Les princesses n'exercent pas de profession d'où tirer leurs moyens de subsistance ni ne gouvernent puisque le «métier de roi » est réservé aux membres masculins de leurs familles. Comme pour ces derniers, les charges domestiques, répondant à leurs besoins physiologiques et matériels, sont exercées par une hiérarchie de subalternes - officiers et domestiques. Ainsi les activités des reines et princesses sont-elles souvent réduites à l'état d'« occupation » - le fait de consacrer son temps à quelque chose - expression au caractère dépréciatif certain.

${ }^{1}$ Ces deux problématiques sont au centre du propos de la somme fondamentale sur l'histoire des femmes: Histoire des femmes en Occident, t. 2, Christiane KLAPISCH-Zuber (dir.), Le Moyen Âge, Paris, Plon, 1991. 
Le rapport au temps est essentiel dans la définition de ces occupations. Une activité devient quotidienne lorsqu'elle est réitérée. Sans préjuger de la fréquence de ce retour, on peut considérer qu'une activité s'inscrit dans le quotidien en raison de son caractère répétitif mais aussi ordinaire et habituel. Les critères qualitatifs qui lui sont attachés sont d'autant plus pertinents lorsqu'on s'attache à un genre social aristocratique - et sexuel - féminin - dont les activités sont socialement, intellectuellement et didactiquement encadrées par des normes juridiques et morales $^{2}$ et par une littérature qui leur propose des archétypes d'emplois du temps souvent éloignés des contraintes de la vie ${ }^{3}$.

Michel de Certeau écrit dans La Prise de parole «qu'il y a histoire quand des groupes ou certains de leurs membres apparaissent comme des acteurs sociaux, c'est-à-dire comme des sujets d'opérations productrices ». Il ajoute : «l'existence sociale d'un groupe relève d'une historicité si par là on entend la capacité qu'a un groupe de se transformer en réemployant à d'autres fins et pour des usages nouveaux les moyens dont il dispose [...]. Un groupe est aussi le sujet de son histoire et pas seulement le produit de contraintes $»^{4}$. Suivant ce raisonnement, la mise en rapport des prescriptions de vie destinées aux femmes de la haute aristocratie et des activités féminines définies comme cause d'un effet -non comme instrument ou conséquence de celui-ci- permet de structurer un premier axe de recherche. Il peut être appliqué aux femmes d'un lignage princier, les duchesses de la seconde Maison d'Anjou (1360-1481). Membres d'une famille « de fleurs de lys » qui dirige une principauté aux possessions territoriales éclatées (Anjou, Provence, Lorraine, Bar), ces femmes sont connues sous le titre de reine de Sicile à partir de

\footnotetext{
${ }^{2}$ Voir la thèse inédite d'Élodie LEQUAIN, L'éducation des femmes de la noblesse en France au Moyen Âge (XIII $-X V^{e}$ siècles), sous la dir. de Colette BEAUnE, Université de Paris-Nanterre, 2005.

${ }^{3}$ Geneviève HASENOHR, «La vie quotidienne de la femme vue par l'Église : l'enseignement des "journées chrétiennes" de la fin du Moyen Âge", dans M. Heinrich ApPELT (éd.), Frau und Spätmittelalterlicher Alltag. Internationaler Kongress Krems an der Donau, 2 bis 5 Oktober 1984, Vienne, Verlag der Österreichischen Akademie der Wissenschaften, 1986, p. 77-100.

${ }^{4}$ Cité par Michelle Perrot, «Une éthique du faire », dans Michel de Certeau. Les chemins de l'histoire, Paris, Complexe, 2002, p. 215.
} 
juillet 1382 - à la suite à l'assassinat de la reine de Naples, Jeanne ${ }^{\text {ère }}$, qui avait adopté Louis $\mathrm{I}^{\text {er }} \mathrm{d}$ 'Anjou, frère du roi de France Charles $\mathrm{V}^{5}$. L'histoire de la Maison d'Anjou se confond alors avec une série de tentatives de conquête du royaume de Naples, héritage convoité. Elle se caractérise aussi par la fréquence - génération après génération - de l'exercice du pouvoir par ses membres féminins.

L'enchaînement des lieutenances générales, exercées du vivant de leur époux, et des régences place les reines de Sicile dans une situation de rupture avec le quotidien d'une reine « ordinaire». Il devient alors légitime de s'interroger sur la divergence que crée une action politique entre les activités quotidiennes des reines la pratiquant ou non. Pour cette étude, le choix a été fait de s'appuyer sur les comptes financiers de la chambre aux deniers et de l'Argenterie des reines de Sicile. Ces sources sont malheureusement lacunaires et n'offrent que des coups de projecteurs sur quelques années. Leur confrontation offre toutefois un échantillon varié sur les différents âges de la vie des reines de Sicile, situées dans des contextes politiques variés. Cette analyse gagne à être complétée par celle de sources narratives, qu'il s'agisse de la littérature prescriptive à destination des femmes de la haute noblesse ou de documents ayant directement trait à la dynastie angevine, ainsi le journal du chancelier de Marie de Blois, Jean le Fèvre (1380-1388) ${ }^{6}$.

\section{La pratique quotidienne de la gestion et de la consommation : tenir son rang de reine}

Les comptes de la chambre aux deniers fournissent des informations sur les recettes, dépenses et dettes de l'hôtel des reines alors que les comptes de l'Argenterie fournissent des détails sur les achats princiers - excepté en matière de

\footnotetext{
${ }^{5}$ Les synthèses principales concernant la seconde Maison d'Anjou sont: Albert LECOY DE LA MARCHE, Le roi René: sa vie, son administration, ses travaux artistiques et littéraires d'après les documents inédits des archives de France et d'Italie, 2 vol., Paris, Librairie Firmin-Didot, 1875 et 1879, et Marcelle REYNAud, Le Temps des princes. Louis II et Louis III d'Anjou-Provence (1384-1434), Lyon, Presses universitaires de Lyon, 2000.

${ }^{6}$ Henri Moranville (éd.), Journal de Jean Le Fèvre, évêque de Chartres, chancelier des rois de Sicile Louis $I^{e r}$ et Louis II d'Anjou, Paris, Picard, 1887.
} 
logement, alimentation et meubles ${ }^{7}$. Si elle permet quelque peu de restituer l'emploi du temps de leurs possesseurs, la comptabilité présente l'inconvénient majeur de ne noter que les activités laissant la trace d'un échange monétaire. En outre, un silence est volontairement maintenu dans ces registres. Tous les rouleaux mensuels de l'Argenterie de Jeanne de Laval commencent ainsi par la mention d'une somme remise :

a nous manuellement comptant pour noz menuz plaisirs et affaires dudit moy la somme de $\mathrm{N}$ l.t. laquelle somme nous avons receue et ne voullons que pour ce il soit tenu rapporter autre quietance, certifficacion ou enseignement que ce present rolles.

La duchesse d'Anjou est avant tout un personnage public qui remplit une fonction de représentation dans le monde curial. Un de ses premiers postes de dépenses concerne les achats de tissus, destinés à son usage propre, à des dons individuels ${ }^{8}$ ou à des distributions collectives, les livrées ${ }^{9}$. Les comptes de l'Argenterie de Jeanne de Laval concernant les années 1456-1459 comprennent une moyenne mensuelle de 121,5 l.t. d'achats de vêtements, dont 27 1.t. pour son propre usage, ce qui représente un quart du total de ses dépenses. Ce pourcentage est

${ }^{7}$ Cette distinction est établie par Maurice REY, Les Finances royales sous Charles VI : les causes du déficit, Paris, S.E.V.P.E.N., 1965, p. 137. L'étude de la comptabilité montre toutefois une réalité plus floue : des mentions de dépenses mobilières se trouvent dans les comptes de l'Argenterie de Jeanne de Laval, les gages des officiers ou la rémunération des messagers se trouvent indifféremment dans l'un ou l'autre des types de comptes. Il semble que la chambre aux deniers résume les dépenses quotidiennes primaires de l'hôtel alors que l'Argenterie comptabilise des dépenses plus ponctuelles (livres, vêtements, joaillerie, jeux, médications...). Il est cependant difficile de dire que l'Argenterie concentre toutes les dépenses extraordinaires des souverains car les comptes de la chambre aux deniers comptabilisent aussi une rubrique sous ce titre.

${ }^{8}$ Nous pouvons noter, en mars 1456, un versement de Jeanne de Laval «a Jehanne Chastellaine, damoiselle de Blanche la Bastarde, la somme de VIII 1. V s.t. pour V aulnes de toille d'atours, qu'elle a baillées et livrées pour ladite Blanche [...], a raison de XXXIII s.t. l'aulne» (Paris, BNF, ms. naf 894, fo4). Entre mars 1456 et février 1457 (datation moderne), elle procède à cinq donations de vêtement à la bâtarde de son époux, ce qui représente $3 \%$ de la dépense totale des vêtements.

${ }^{9}$ Jeanne de Laval écrit ainsi le 30 septembre 1479 une lettre-patente à destination des gens de la chambre des comptes dans laquelle elle certifie avoir dépensé 243 florins 9 gros, « laquelle somme il a paie et baillee et delivree aux dames et damoiselles et femmes de chambre de nostre hostel pour leurs robes delivrees de l'annee finye au derrain jour de septembre derrain passé » (Aix-enProvence, AD des Bouches-du-Rhône [BDR], B. 2510, f²4v). 
identique pour la période $1479-1480^{10}$. Les sommes consacrées à la joaillerie et à la tapisserie sont également importantes, destinées à un emploi personnel ou à participer à l'économie du "don en représentation $»^{11}$, particulièrement durant la période des étrennes - nécessaire à l'entretien des relations avec les autres cours ${ }^{12}$.

Ces achats s'effectuent par l'intermédiaire d'un officier mandaté directement par la reine, selon une formule récurrente retranscrite dans l'exemple suivant :

de nostre ordonnance et commandement ont esté pris et acheté de Jehan Nepveu, marchant de nostre cité, a faire robe a Mariolle, bastarde de Chastillon, touteffois et quantes que sera nostre plaisir, et d'iceulx draps avons fait le pris et marché [...] par le sire des Roches, nostre maistre d'ostel, a ladite somme de IIIIXX XI florins ${ }^{13}$.

À une époque où le vêtement ne s'achetait pas d'un seul tenant mais était composé de différents éléments, la reine choisissait le type et la valeur du tissu. Elle déterminait également son emploi dans le vêtement. Jean de Bar, son pelletier, s'est ainsi fait rembourser en avril 1456 :

[...] pour XXXVI peaux de menu ver qu'il a mis et emploiez par nostre commandement et ordonnance en ung collet, unes choistes et ung ougnetz pour Michou la Folle. Vallent les XXXVI peaux [...] la somme de XVIII l.t. ${ }^{14}$.

${ }^{10}$ Les calculs sur le premier registre (Bibl. mun. d'Angers, ms. 1064) ont été effectués par Françoise PIPONNIER, Costume et vie sociale: la cour d'Anjou (XIV -XV siècles), Paris-La Haye, EPHE et Mouton \& Cie, 1970, p. 97. La conversion est effectuée selon la valeur de l'écu neuf à la couronne crée par Charles VII qui valait 1,5 l.t. dans les années 1450. Cette opération a été reproduite pour l'étude du manuscrit AD BDR, B 2510.

${ }^{11}$ La pratique du don et du contre-don est mise en évidence dans cet extrait : «A luy pour II aulnes de veloux noir qu'il a baillé et livré par nostre commandement au sire de Veslins, serviteur de nostre belle mère de Laval, lequel nous a présenté de par nostre mere ung bel esturgeons et ouquel sire de Velins nous avons donné ledit veloux pour cecy a raison de III escuz et quart l'aulne, des escuz a preseng ayans court, VI escuz et demy valent VIII 1. XVIII s. IX d.» (BNF, ms. naf 894, f $10 \mathrm{v})$.

${ }^{12}$ Voir l'article de Françoise RoBIN, «Jeanne de Laval et ses orfèvres : étrennes et présents à la cour d'Anjou-Sicile (1456-1458)", dans Reines et princesses an Moyen Áge, Actes du cinquième colloque international de Montpellier, Université Paul-Valéry (24-27 nov. 1999), t. 2, Montpellier, Cahiers du CRISIMA, 2001, p. 113-125.

${ }^{13}$ AD BDR, ms. 2510, fo26.

${ }^{14} \mathrm{BNF}$, ms. naf 894, f 14 . 
La reine de Sicile agit comme une maitresse de maison. Ses choix provoquent les actions des officiers à son service. Le temps de sa réflexion et de sa prise de décision, qui engendre sa délégation et donc l'action de ses serviteurs - c'est-à-dire l'achat et la confection d'un vêtement - incite à considérer comme une activité quotidienne aristocratique féminine l'acquisition et la confection vestimentaire. Nous ne possédons malheureusement que les comptes de l'Argenterie de Jeanne de Laval. Le premier décrit la culture matérielle et les activités d'une jeune mariée; le second ceux d'une reine vieillissante, retirée en Provence aux côtés de son époux. Il serait fécond de les comparer avec les registres concernant des femmes actives en politique. Un cas intéressant permet d'extrapoler à ce sujet.

Isabelle de Lorraine, envoyée à Naples en 1435 par son époux René d'Anjou - prisonnier du duc de Bourgogne depuis $1431^{15}$ - pour garder la ville contre son compétiteur, Alphonse V d'Aragon, fut contrainte de vendre ses joyaux et autres objets d'orfèvrerie pour financer à la fois son hôtel et le coût de la guerre menée par l'État angevin. Une lettre-patente, instituant Conrad Parsperguet comme nouveau maitre de ses finances, résume un de ses comptes, établi entre le $1^{\text {er }}$ octobre 1436 et le 31 avril 1438. Il permet de connaitre la teneur et le montant des ventes d'objets précieux auxquelles procède la reine de Sicile ${ }^{16}$. La somme qui en résulte représente $29 \%$ du total de la recette. Parallèlement, la reine ne finance que deux dons individuels de vêtement. L'activité somptuaire n'est plus prioritaire pour une femme qui exerce le pouvoir dans une contrée lointaine et dans des conditions de précarité visible. Réorientation des priorités ne signifie pas abandon de l'intérêt pour ces objets précieux : $72 \%$ des dépenses extraordinaires (comptabilisées hors du coût quotidien de son hôtel), soit $18 \%$ du total de ses dépenses, sont consacrés aux remboursements des officiers qui vont « desgaiger» des objets que la reine de Sicile avait mis en gage pour se procurer des liquidités.

${ }^{15}$ Voir Bertrand SCHNERB, Bulgnéville (1431). L'État bourguignon prend pied en Lorraine, Paris, Economica, 1993.

${ }^{16}$ Paris, BNF, ms. coll. lorraine 20 bis. 
Cet exemple permet d'infléchir une impression consumériste fréquente à la lecture des comptabilités princières. Il faut rappeler que la consommation des duchesses angevines correspond à la mise en pratique d'activités conformes au code de conduite attendu des aristocrates. Les comptes de l'Argenterie de René d'Anjou sont également surchargés de distributions vestimentaires en tout genre ${ }^{17}$. Un des plus intéressants «miroirs de princesses » de la fin du Moyen Âge, rédigé par une femme de pouvoir, les Enseignements d'Anne de France à sa fille Suzanne, future duchesse de Bourbon (1505), permet de mieux comprendre ces enjeux financiers. Selon Éliane Viennot, les Enseignements livrent la clef d'une carrière politique féminine placée sous le signe d'une dissimulation qui se confondait avec l'apparence d'une soumission féminine ${ }^{18}$. Dans le chapitre «touchant ces habits et atours », Anne de France préconise à sa fille de «toujours tant que vous habilliez le mieux et plus nettement que pourrez. Car au regard du monde, croyez pour vrai qu'il est malséant et fort déshonnête de voir une fille ou femme noble nicement habillée et mal en point ». Ce conseil est d'autant plus pressant que ce sont les «nobles femmes, qui, voluntiers, sont plus regardées que les autres » et qui jouent le rôle de « miroer patron et exemple des autres $»^{19}$.

Dans un autre chapitre, Anne de France rappelle l'importance de l'éducation morale et religieuse qu'une princesse doit dispenser à ses futurs enfants - éducation qui passe avant tout par l'exercice de ses propres vertus dans ces domaines. La comptabilité permet d'appréhender la pratique religieuse ordinaire des duchesses d'Anjou. Les aumônes sont systématiquement mentionnées au début de chaque rouleau de l'Argenterie de Jeanne de Laval, avec le même montant mensuel4 livres tournois dans les années 1450 et 12 florins dans les années 1480-, faibles

\footnotetext{
${ }^{17}$ Voir leur édition par l'abbé Arnaud D’AGNEL, Les Comptes du roi René publiés d'après les originaux inédits conservés aux archives des Bouches-du-Rhône, 3 vol., Paris, 1908.

${ }^{18}$ Éliane Viennot et Tatiana Clavier (éd.), Anne de France. Enseignements à sa fille, Saint-Étienne, Publications de l'université de Saint-Étienne, 2006, p. 47.

${ }^{19} I d$., p. 47 et 65 . Sur le rôle de la reine comme « corps de mode», voir Rachel GIBBONS, «The Queen as 'social mannequin'. Consumerism and Expenditure at the Court of Isabeau of Bavaria (1393-1422) », Journal of Medieval History, 26/4, 2000, p. 371-395.
} 
montants au regard des dépenses vestimentaires. Outre l'institution de l'aumônerie, la charité peut être ponctuelle : revient à intervalles réguliers la mention de sommes destinées aux pauvres, aux ermites et aux églises desservant la messe pour les duchesses ${ }^{20}$. Les dépenses extraordinaires sont rares comme en témoigne l'unique fondation par Isabelle de Lorraine d'une chapelle au sein de sa cour. La lettrepatente instituant les moyens de rétributions de ses chapelains et de ses chantres les destinent à lui assurer des offices spirituels journaliers ${ }^{21}$. Assurer le service quotidien de son âme est une préoccupation majeure et, dans le cas d'Isabelle de Lorraine, elle s'est traduite par une attention particulière portée aux chantres, donc à la musique sacrée.

L'activité pieuse des reines participe à leur rôle d'éducatrice morale et religieuse à la cour. Les comptes de la chambre aux deniers de Marie de Blois et Yolande d'Aragon permettent de prendre la mesure de la dimension concrète de cette éducation $^{22}$. Yolande d'Aragon, de février 1409 à septembre 1414, soit cinq ans et sept mois, ne reste séparée de sa progéniture que trois mois et vingt-trois jours, soit 4,8\% de son temps ${ }^{23}$. Dans le compte suivant (septembre 1414décembre 1418), les séparations deviennent plus fréquentes au fur et à mesure que les enfants grandissent. La plus importante s'étend de mars 1416 à janvier 1417, soit

${ }^{20}$ Les sept mois du voyage de Marie de Blois la conduisant d'Anjou en Languedoc sont rythmés par les offrandes qui accompagnent son assistance aux offices : douze mentions sont notées à ce propos dans le compte $\mathrm{AN}$, KK. 241 et elles représentent $8 \%$ de ses dépenses extraordinaires.

${ }^{21}$ AN KK 245 f $1-1 v$. Il faut souligner que la chapelle est fondée conjointement par Isabelle de Lorraine et René d'Anjou en mai 1449. Or, contrairement à l'usage, leurs secrétaires rédigent deux lettres-patentes autonomes mettant en valeur le rôle moteur de la reine dans cette création, seule à lui affecter des revenus dès mai 1449 : «Comme monseigneur, pour aider a supportez noz affaires, nous eust despiecaz donné [...] le revenu, prouffit et emolument des tabliers de nostre dit pais de Prouvence [...] pour bon regart ait voulu et nous semblablement iceulx deniers estre receuz et emploiez ou payment de certain nombre de bons chappelains et chantres qui doresenavant chascun jour feront beaux services devant mondit seigneur et nous ou l'un de nous en l'absence de l'autre, pour lesquelx deniers recevoir [...]». René d'Anjou se contente de confirmer ces dispositions.

${ }^{22}$ Nicholas Orme, From Childhood to Chivalry. The Education of English Kings and Aristocracy (10661530), Londres, 1984, souligne que l'éducation de la jeunesse est primordiale pour la prospérité de la maison princière et que les reines sont mises à contribution pour l'assurer.

${ }^{23} \mathrm{AN}, \mathrm{KK} 243$ est un registre qui contient plusieurs comptes de la chambre aux deniers de Yolande d'Aragon. La datation utilisée est modernisée. 
9 mois. Yolande d'Aragon seconde son mari à Paris et laisse ses enfants à Angers pour les protéger du conflit qui sévit dans la capitale entre Armagnacs et Bourguignons. Peu à peu, l'héritier commence à quitter ponctuellement le giron maternel pour recevoir une éducation politique que sa mère n'est pas chargée de lui fournir. Différents référents masculins - connus par leurs rétributions - sont chargés de suppléer l'absence du duc d'Anjou, décédé en avril $1417^{24}$. La comptabilité témoigne d'une répartition sexuelle des tâches princières selon laquelle l'activité maternelle incombe aux reines de Sicile: les dépenses des enfants de la cour sont toujours prises en charge par ces femmes, qu'elles se trouvent ou non avec eux, et ce, même si les enfants ne sont pas les leurs ${ }^{25}$. Toutefois, l'importance de l'activité domestique ne réduit pas l'horizon féminin aristocratique - tant géographique que relationnel - à celui de son hôtel.

\section{L'espace ouvert aux activités des duchesses d'Anjou}

Entretenir les relations avec les membres de son entourage, donc les fidélités, comme l'écrit Anne de France, est essentiel : «[...] en leurs gésines, fortunes, maladies, vous devez visiter, et envoyez de votre hôtel quelque chose de nouveau, si vous l'avez, que vous pensez que leur soit nécessaire ou plaisant». Encore une

\footnotetext{
${ }^{24}$ Par exemple AN KK 243, $\mathrm{f}^{\circ} 49 \mathrm{v}$ : «A Antonnet Armentier cappittaine de trois cens hommes d'armes et cent arbalestriers pour la paye de lui et eulx pour un mois commancant le XVII ${ }^{\mathrm{e}}$ jour dudit moys d'avril VIII ${ }^{\mathrm{C}}$ III l.t, lesquelx cent arbalestriers les compte en la compaignie dudit Anthonnet ont esté ou service de monseigneur d'Anjou en son dit voiage et les autres comptes es garnisons du Mans et de la Ferté; a monseigneur l'evesque d'Angers pour son estat et ordonnance d'un mois faisant le voyage [...], a raison de VIII 1. par jour, $\mathrm{II}^{\mathrm{C}} \mathrm{XL}$ 1. ; au juge d'Anjou pour semblable, a LX 1. par jour, IIII ${ }^{\mathrm{XX}} \mathrm{X}$ 1. ; a Jehan Dupuy pour semblable IIII ${ }^{\mathrm{XX}} \mathrm{X} 1$. ; a Jehan Porchier pour semblable, a XL 1. par jour, LX 1. ; [...] a monseigneur d'Anjou que la royne lui a ordonné es moys de mars et avril pour fere a son plaisir XLV 1., lesquelles sommes montent en tout XIII ${ }^{\mathrm{C}} \mathrm{IIII}^{\mathrm{XX}}$ XV 1. X s.t. Donné le XII ${ }^{\mathrm{e}}$ jour de may 1418 ».

${ }^{25}$ Le registre AN KK 243 décrit les dépenses de l'hôtel de Yolande d'Aragon comme celles « de Madame et de ses enfans ». La duchesse prend en charge les dépenses du comte de Ponthieu, futur Charles VII, fiancé de sa fille Marie et de Catherine de Bourgogne, fiancée du comte de Guise, futur Louis III d'Anjou. Il importe de souligner la symbolique de cette répartition financière entre le duc et la duchesse d'Anjou, non son coût, car le trésorier d'Isabeau de Bavière, Hémon Raguier, rembourse les frais de l'hôtel de son fils à Yolande d'Aragon (AN, KK. 243, $\left.\mathrm{f}^{\circ} 13\right)$.
} 
fois, l'Argenterie de Jeanne de Laval est riche en renseignements. L'ensemble des frais consacrés à ses chariots et attelages (qui montent à $25 \%$ de ses dépenses hors mois d'étrennes) résulte de l'importance accordée aux déplacements. Ils lui permettent notamment de respecter le précepte d'Anne de France en se rendant par exemple :

a Monstereul Bellay pour veoir nostre belle seur de Tancarville, estant en couche lors de nostredite niepce ${ }^{26}$.

Certains de leurs officiers peuvent aussi effectuer leurs voyages par procuration, ainsi le confesseur de Jeanne de Laval, Olivier de Pennart, qui reçoit :

XI l.t. en VIII escuz d'or [...] pour ung voiaige par luy fait par nostre commandement [...] ou dit moys de janvier derrain passé, partant de nostredite ville d'Angier a Redon devers nostre bel oncle le duc de Bretaigne, et a Laval devers nostre belle mère, pour certains noz affaires, ou voiaige il a vacqué par l'espace de huit jours entiers ${ }^{27}$.

Ces déplacements ne correspondent pas à des voyages d'agrément contrairement à la pratique de monter à cheval qui relève des loisirs féminins princiers, parmi lesquels l'assistance aux farces et l'écoute de musique sacrée ou profane $^{28}$. Ordinairement, les comptes de la chambre aux deniers n'évoquent que les points de départ et d'arrivée des reines - le plus souvent entre les pôles structurants de la principauté, l'Anjou et la Provence. Un des livres de Marie de Blois permet toutefois de retracer avec précision son itinéraire car, brûlé en 1373 par les Anglais au fort de Vaas où il était conservé, il a été reconstitué à partir des écrous des six services de son hôtel du 13 juillet 1365 au 7 mars $1366^{29}$. Durant sept mois, l'itinérance rythme sa vie, la conduisant de Saumur à Béziers, cheminant par la Touraine, l'Anjou, l'Orléanais, la Bourgogne, le Lyonnais, la Provence avant

\footnotetext{
${ }^{26} \mathrm{BNF}, \mathrm{ms}$. naf $894, \mathrm{f}^{\circ} 14$.

${ }^{27}$ Ibid., f f $^{3} 8$.

${ }^{28}$ Jeanne de Laval achète deux selles, une pour sa belle-fille Yolande d'Anjou et l'autre pour sa propre haquenée (BNF, ms. naf 894, f $\mathrm{f}^{\circ}$ 50). Les autres loisirs curiaux féminins sont connus par des dons ponctuels et personnels accordés par les duchesses, qui ne rémunèrent pas de personnel à des fins de distraction.

${ }^{29}$ Paris, AN. KK. 241. La datation est modernisée.
} 
d'arriver en Languedoc. Son époux n'est pas à ses côtés puisqu'il ne prend pas en charge ses finances, ce qu'il fait systématiquement lors des épisodes de vie commune. Cette pratique, courante au sein des maisons princières, «virilise » le temps passé en présence de l'homme qui montre sa capacité à entretenir sa famille. Cette manifestation de domination masculine est d'autant plus manifeste que l'on retrouve des mentions de remboursement de frais avancés, en leur absence, par les ducs à leurs femmes. En mars 1405, Yolande d'Aragon rembourse ainsi Louis II pour avoir réglé les frais, par l'intermédiaire de son maittre d'hôtel, de son voyage d'Anjou en Provence ${ }^{30}$. Cette mention est d'autant plus étonnante que le même compte nous apprend qu'une fois arrivée «en pays de par-deçà » où elle retrouve son époux, ce dernier reprend en charge la duchesse.

Le prince angevin influe également sur l'itinéraire de son épouse. En 1365, le trajet de Marie de Blois est déterminé par les activités de Louis ${ }^{\text {er }}$. Elle le retrouve à la cour du pape d'Avignon avant de se rendre en Languedoc, province dont il devient le lieutenant général de Charles V en juin 1365. Une fois sortie de l'apanage, elle se déplace presque quotidiennement et parcourt en moyenne vingt kilomètres par jour. Son cortège - comprenant des gens d'armes destinés à assurer sa sécurité - s'arrête tous les midis pour déjeuner et gît la nuit chez des hôtes. Certaines haltes peuvent durer plusieurs jours et s'accompagnent de déplacements à l'échelle locale. Nous ignorons en général la raison de ces rayonnements régionaux - excepté dans le cas d'un pèlerinage à «l'île de la Barbe » au nord de Lyon le 4 décembre 1365. Une des seules activités constantes documentée concerne sa pratique religieuse $^{31}$. À partir de l'arrivée sur le Rhône, les déplacements de Marie

\footnotetext{
${ }^{30} \mathrm{Un}$ fragment de compte de Louis II d'Anjou nous apprend, dans le cadre de ses recettes, qu'il reçut: "le IX ${ }^{\mathrm{e}}$ jour dudit moys de mars [1405] des coffres de la royne par la main de Colaz Joullain en la présence de Jehan Deni, maistre d'ostel dudit seigneur, pour partie de sa despence par XLII jour que elle mist à venir d'Anjou à Tharascon et que elle fut à sa despence, le roy estant en Aix, sa dicte despence comptée en la chambre aux deniers du Roy. Pour ce mil VI ${ }^{\mathrm{C}}$ LXX 1. VIII s. VIII d.», dans Paul BONENFANT (éd.), "Fragment de comptes de Louis II d'AnjouProvence, roi de Naples (1405) », dans Revue belge de philologie et d'histoire, t. III, 1924, p. 848.

${ }^{31}$ Infra, note XX.
} 
de Blois s'effectuent par voie d'eau, non plus par voie de terre, et constituent la dernière étape du voyage, brièvement interrompu par un arrêt mensuel à Viviers, ville royale du Vivarais (Ardèche). La duchesse y retrouve les activités quotidiennes détaillées au début de cette étude.

Les circuits des coursiers témoignent de l'active correspondance entretenue par les duchesses, tant dans leur rôle d'épouse que de femme de pouvoir, élargissant l'horizon de leur influence. Jeanne de Laval, qui n'exerce jamais officiellement de pouvoir politique, tente d'influer auprès du pape pour défendre un archevêque angevin en $1479^{32}$. Avant son arrivée en Avignon en 1365, Marie de Blois écrit fréquemment à son mari. Une fois veuve, son chancelier, Jean le Fèvre, fait état de l'importance de son courrier. Il est difficile de connaittre la fréquence et la teneur de ces documents épistolaires. Certains possèdent des portées véritablement politiques, comme celles que Marie de Blois adresse aux oncles de Charles VI ; une portée administrative, comme celles qu'elle envoie à ses receveurs et trésoriers; une portée privée dont le but est surtout d'entretenir des relations sociales. Cette visée est au cœur d'une missive envoyée par Isabelle de Lorraine, alors isolée à Naples, à son beau-frère, le marquis de Bade :

Treschier et tresamé frere, pour ce que de tout mon cuer desire savoir le certain du bon estat et santé de vostre personne, lequel Nostre Seigneur vueille faire tel et si bon comme vostre cuer le desire et que pour moy le vouldroye [...]. Je tieng que pareillement estez desirant savoir j'estoie [par] l'escripture de cestes en bonne santé de ma personne, mercy Nostre Seigneur qui vous ottroit, treschier et tresamé frere, quant a l'estat et besoignes de par deça ensemble toutes nouvelles par mez amez, les menestrelz de monseigneur, qui a present s'en retournent par dela, en pourez s'il vous plait estre a plain acertenez. Au surplus, je vous prie tant comme je puis que de voz nouveles me vueillez rescripre avec aucune chose si voulez par deca que faire puisse, et de bon cuer je l'acomplirai a mon pouvoir. Au plaisir de Nostre Seigneur qui vous ait en sa sainte garde. Escript a Neapoli le XXII jour de fevrier.

${ }^{32}$ «Au valet de pied Colinet pour sa dépense et celle d'un cheval pour estre aller 3 jours à Avignon porter des lettres à Loys Danoys pour envoyer à Rome pour un courrier par les affaires de Beaufroit l'archevêque - 1 florin, 6 gros » (AD BDR, ms. 2510, f ${ }^{\circ}$ 9). 
YZABEL, royne de Jherusalem et de Sicile, duchesse d'Anjou, de Bar et de Lorraine et contesse de Prouvence ${ }^{33}$.

Ce document épistolaire use des formules propres aux lettres closes familières : un style plus libre que celui des lettres-patentes visible dans l'absence de titulature officielle du destinateur inaugurant son contenu, une apostrophe familiale, l'introduction de l'expression « escript à » au lieu de «donné à » ou « la reine en son conseil» et l'absence de datation annuelle. Les grandes aristocrates sont prédisposées à l'entretien des relations sociales nécessaires aux appuis politiques. Les princesses y participent au profit de leur époux et peuvent même désamorcer les erreurs de ce dernier par leur inlassable activité sociale, comme le préconise Anne de France :

Et semblablement, devriez mettre peine plus qu'onques mais d'acquérir l'amour de ses seigneurs et amis [...] pour échever les mauvais jugements (qui, à cause du déshonnête gouvernement des fols maris, sans cause se font sur les femmes souvent), comme pour plus fort complaire à votre mari, pour le retraire et acquérir son amour, et [celui] de ses amis.

Ces rapports socio-politiques sont d'autant plus importants dans le cadre d'un veuvage ou d'une lieutenance, puisqu'elle ajoute :

Et du gouvernement de leurs terres et besognes ne s'en doivent attendre qu'à elles, touchant la souveraineté, ni n'en doivent donner puissance à nul qui soit. Et alors, vous devez vous garder d'avoir serviteurs trompeurs ni cuideraux, en espécial ceux qui souvent ont à besogner, pour les charges qui s'en peuvent suivre, tant sur vous que sur vos filles, soient les serviteurs, parents, ou autres $[\ldots] .{ }^{34}$

Le soutien d'hommes fiables est nécessaire à la gestion financière que l'analyse de la comptabilité des reines de Sicile permet, en dernier ressort, d'étudier.

\section{L'activité financière des reines de Sicile}

\footnotetext{
${ }^{33}$ Paris, BNF, coll. Lorr., 20 bis, $\mathrm{n}^{\circ} 7$.

${ }^{34}$ Éliane Viennot et Tatiana Clavier (éd.), op. cit., p. 87.
} 
Selon Christine de Pizan, les femmes de l'aristocratie n'ont pas l'habitude de s'occuper des comptes de leur maison, situation qu'elle réprouve :

Le .VII e. enseignement de Prudence a la sage princepce est qu'elle prendra garde soingneusement au fait de sa revenue et de sa despence [...], n'aura point de honte elle meismes de vouloir savoir la somme de ses revenues ou de ses pensions, ne que les comptes de ses receveurs et despensiers de ses finances soient a certains jours fais devant elle ${ }^{35}$.

Or, nous pouvons remarquer l'attention que les reines de Sicile apportent à l'établissement de leurs comptes. Jeanne de Laval, dans son Argenterie des années 1479-1480, ne laisse pas de doute à ce sujet. Elle conclut chaque dépense mensuelle en s'adressant aux membres de la chambre des comptes comme suit:

... lesquelles parties cy dessus declayrons nous avons ouy lire de mot a mot devant nous et les gens de nostre conseil et les avons trouvees vrayes et bien recolantes et memoratives, et pour ce qu'en voullons nostredit argentier estre et demourer quicte et deschargié comme raison est [...].

La reine de Sicile ne délègue pas sans contrôle ses finances. Rien ne l'y oblige puisque les gens de la chambre des comptes sont préposés à leur vérification. Il s'agit donc d'une activité volontairement choisie, d'une responsabilité exercée au sein d'un conseil. Ni veuve ni lieutenante, l'usage du terme de «conseil» pour désigner ses officiers domestiques recouvre sans doute, plus qu'une réalité politique, la conscience qu'a Jeanne de Laval d'exercer un pouvoir de gestion à une échelle mineure. Nous savons que René d'Anjou lui avait, de son vivant, cédé en pleine propriété le comté de Beaufort, au détriment de la coutume angevine l'autorisant à administrer les biens de sa femme. Cette dernière rejoint par la pratique financière ses aïeules, poussées à l'autonomie par des circonstances bien plus dramatiques.

Une femme à la tête d'une principauté se trouve confrontée aux limites, tant juridiques que mentales, attachées à son genre, mais surtout aux propres difficultés financières du territoire à gérer. Dans le cas de la seconde Maison d'Anjou, ces

${ }^{35}$ Christine de Pizan, Le Livre des trois vertus, Charity Cannon Willard et Eric Hicks (éd.), Paris, Champion, 1989, p. 74-75. 
problèmes sont aggravés par les entreprises napolitaines de la dynastie. Arrivées au pouvoir de façon fortuite, les reines de Sicile doivent s'approprier les moyens de gouvernement élaborés pour leurs époux. Elles procèdent souvent à une concentration des différentes comptabilités, toujours confiées à des officiers domestiques déjà à leur service du vivant de leur mari ${ }^{36}$. Arrivée en Provence en 1385 - à charge pour elle de conquérir l'héritage de Jeanne İ̀re de Naples - Marie de Blois, veuve, ouvre par une lettre-patente un compte où elle se présente

[...] ayant le bail, garde et administracion de Loys, roy desdits royaumes, et Charles, noz enffanz maindres d'aage, et de toutes leurs terres [...]. Nous avons [...] besoing avoir prestement de deniers desquels fere finance. Nous envoions en nos pais de France nostre amé et feal grant maistre d'ostel, messire Jehan Pelerin, et en sa compagnie nostre bien amé maistre, Jehan Vié, maistre de la chambre aux deniers du roy nostredit filz pour ceux deniers recueillir et recevoir et nous apporter ou envoier. Et pour ce que nous, confians de la loyauté et bonne diligence dudit maistre Jehan, luy avons donné et donnons par ces présentes lectres et mandement especial de recevoir iceulx deniers pour nous et en nostre nom, soit de noz receveurs et sergenz, comme de quelzconques autres $[\ldots]^{37}$.

Autrement dit, Marie de Blois recherche des liquidités en envoyant ses officiers domestiques prélever des sommes sur tous les receveurs de l'apanage angevin - prélèvement que seul le trésorier est normalement habilité à faire. Cet argent est en partie employé à soutenir l'entreprise militaire napolitaine, à hauteur de 38900 francs - soit 38900 l.t. puisqu'en 1385, un franc or équivaut à 20 s.t. Cette somme représente $64 \%$ de ses dépenses et ne recouvre que les frais « réels »

\footnotetext{
${ }^{36}$ Voir le compte de Marie de Blois (BNF, ms. fr. 11 863) qui rassemble les caractéristiques d'un compte de trésorerie sans jamais en porter le nom ni qu'un trésorier ne se charge de sa gestion. Le journal de Jean le Fèvre apporte des renseignements sur le rôle joué par le maître de la chambre aux deniers de Marie, Pierre Payen, qui dépasse largement les attributions habituelles de son office. Il écrit par exemple : «... du premier jour de May l'an MCCCIIII ${ }^{\mathrm{XX}}$ et V jusques au premier jour de janvier ycellui an, par le compte de maistre P. Payen la guerre de Prouvence a cousté à Madame XXXI ${ }^{\mathrm{M}} \mathrm{III}^{\mathrm{C}}$ LI frans », dans Henri MORANVILLE (éd.), op. cit., p. 301. La lettrepatente d'Isabelle de Lorraine montre le même souci d'efficacité en rassemblant toutes les comptabilités en une (BNF, coll. Lorr., 20 bis, $\mathrm{n}^{\circ} 7$ ). Ce compte résume les dépenses de son hôtel, les dépenses de guerre et les quelques rares dons dont il a été fait mention précédemment, tous gérés par Conrad Parsperguet, son maitre d'hôtel.

${ }^{37}$ Paris, BNF, ms. fr. 11 863, f $37 \mathrm{v}$.
} 
de la guerre, sans compter les frais occasionnés par la recherche de fonds (envoi de messagers, d'ambassadeurs, frais de crédit et de change...), par exemple :

[...] messire Jehan de Beaumal pour porter en Ytalie aux soudayers [...]

[...] pour le fait de l'armée de la mer pour envoiez oudit royaume de Sicile [...]

[...] pour porter en Ytalie pour les gaiges des capitaines et gens d'armes estans en la conqueste du royaume de Sicile [...]

Si des officiers sont préposés à s'occuper des modalités techniques de la comptabilité (rédaction des mandements pécuniaires et des registres de comptes, vérification de leur validité) et si des conseillers jouent un rôle essentiel dans la stratégie - non pas budgétaire à une époque où la notion de budget est encore inconnue, mais plutôt pécuniaire-, les reines de Sicile conservent une réelle autonomie de décision en ce domaine. Il s'agit, d'un point de vue socio-politique, de négocier des prêts avec d'autres puissances - notamment la papauté et la couronne de France -, mais aussi de se garantir la fidélité des villes et des seigneurs fieffés dans la principauté pour leur imposer un «prêt forcé » ou tout simplement de réussir à accroître les prélèvements fiscaux dans le domaine ducal. Le journal du chancelier de Marie de Blois, Jean le Fèvre, rapporte comment l'autorité de celle-ci s'applique aux stratégies financières, par exemple dans la fixation du montant des gages :

Apres disner je fis rassambler le conseil en la chambre à l'instance messire J. de Bueil qui [...] requeroit avoir meilleurs lettres [de gages] : par le conseil fut la chose remise à Madame et je me chargé de li en parler' ${ }^{38}$.

La vente des objets précieux par Isabelle de Lorraine, déjà évoquée, est destinée à la construction d'une bombarde et à la solde de mercenaires génois engagés pour combattre sur mer. Répondant aux mêmes nécessités belliqueuses souvent minorées chez les historiens qui, à la suite des lettrés du Moyen Âge, considèrent la femme de l'aristocratie avant tout comme un agent de paix-

\footnotetext{
${ }^{38}$ Journal de Jean le Fèrre, op. cit., p. 338. Mention datée du 15 mars 1387.
} 
Yolande d'Aragon choisit des mesures d'économie drastiques. Le décès de son époux impliquant le transfert des officiers de ce dernier dans son propre hôtel, elle interdit dès septembre 1417 à tous ses serviteurs d'y être automatiquement nourris et vêtus en contrepartie du versement de gages fixes, limitant les abus et permettant un meilleur contrôle du coût de l'hôtel. Cette mesure est unique dans l'histoire financière de la maison d'Anjou. Le seul autre prince à la mettre en œuvre est Charles le Téméraire, cinquante ans plus tard. Yolande d'Aragon verse également d'importantes sommes de ses propres coffres. Dans son testament, elle avoue, afin de justifier la pauvreté de ses legs, que :

Item pour ce que par aventure aucuns pourroient a nous en ymaginacion consideré la quantité de meuble tant d'or, d'argent, vesselle royaux et autres biens et choses que nous demourerent apres le decees de nostredit feu seigneur et espoux, et encore en deussions avoir en grant nombre, nous disons et declairons pour rendre contens ceulx qui en pourroient doubter, que tout le plus bel et le meilleur a esté employé pour le fait du royaulme d'Italie et baillé au roy Loys nostre ainsné filz, dont Dieu ait l'ame, pour sa conqueste; autre partie cy acquict de doibtes de nostredit feu seigneur et espoux dont nous demourasmes chargié, et aussi en avons mis grant nombre pour la deffence du pays durant que avons eu le bail de noz enfans, et de present n'avons autres biens meubles que ceulx que avons monstré et baillé par inventaire a nostredit filz Charles ${ }^{39}$.

Yolande d'Aragon, tout comme les autres femmes de pouvoir de sa dynastie, a négligé ses activités somptuaires et sacrifié ses finances à la conquête napolitaine, entamée et poursuivie par les hommes.

Initiées aux finances par la gestion de leur douaire - activité quotidienne «ordinaire » de toute princesse, les reines de Sicile se révèlent en définitive aussi actives que leurs époux dans celles de la principauté. Cette activité, « extraordinaire» au regard de leur condition de femme, devient quotidienne. La comparaison de leurs registres comptables à ceux des hommes de la dynastie

${ }^{39}$ AN, P $1334 / 17, n^{\circ} 52$, fol. XXI. 
angevine fait apparaître une manière originale de gérer ces finances «semipubliques ». La comptabilité représente une source intéressante pour appréhender la vie des Grandes. Les informations qu'elle délivre ne sont connues ni à travers un prisme masculin, ni à travers des prescriptions comportementales. Elle renvoie à un contenu qui se veut objectif en recensant tous les actes ayant suscité un échange monétaire - avec toutes les limites que ce critère impose. L'accent porte sur des choix liés à la représentation, dont le coût correspond à l'autocélébration attendue de la haute aristocratie. Les correspondances entre les activités des duchesses d'Anjou et celles préconisées par les «miroirs de princesse », reflétant les valeurs projetées sur le genre féminin, sont visibles. L'autonomie et l'écart à la norme sont toujours possibles et s'attacher aux finances permet d'appréhender certaines actions méconnues des femmes de pouvoir, ainsi leur participation à la logistique guerrière de la principauté. Restituer le vécu des femmes (et des hommes) - fantasme ultime de l'historien - ne peut toutefois être que partiel, même si l'analyse comptable permet d'observer un de ses reflets les plus fidèles. 